\title{
Estudo do crescimento relativo de Austinixa patagoniensis (Rathbun) (Decapoda, Pinnotheridae) simbionte de Callichirus major (Say) (Decapoda, Callianassidae) no mesolitoral da praia de Balneário Camboriú, Santa Catarina, Brasil
}

\author{
Eliana dos S. Alves ${ }^{1}$, Sérgio de A. Rodrigues ${ }^{2} \&$ Paulo R. Pezzuto ${ }^{3,4}$ \\ 1 Programa de pós-graduação em Ecologia, Instituto de Biociências, Universidade de São Paulo. Rua do Matão, Travessa 14, \\ 321, 05508-900, São Paulo, E-mail: lialves@lycos.com \\ ${ }^{2}$ Departamento de Ecologia, Instituto de Biociências, Universidade de São Paulo, São Paulo. In memoriam. \\ ${ }^{3}$ Centro de Ciências Tecnológicas da Terra e do Mar, Universidade do Vale do Itajaí, Rua Uruguai 458, 88302-202, Itajaí, \\ Santa Catarina, E-mail: pezzuto@univali.br \\ ${ }^{4}$ Autor correspondente.
}

\begin{abstract}
Relative growth of Austinixa patagoniensis (Rathbun) (Decapoda, Pinnotheridae), a symbiotic crab of Callichirus major (Say) (Decapoda, Callianassidae) on the midlittoral of Balneário Camboriú beach, Santa Catarina, Brazil. The relative growth of Austinixa patagoniensis (Rathbun, 1918), a symbiotic crab inhabiting burrows of the callianassid shrimp Callichirus major (Say, 1818) in Balneário Camboriú beach, Santa Catarina (26 $59^{\circ} \mathrm{S}, 48^{\circ} 39^{\prime} \mathrm{W}$ ) was studied in 119 juveniles, 228 males and 249 females. Functional regressions were used to describe the relative growth of the following variables: carapace length and width, abdomen width, maximum height and length of the upper and lower margins of the propodus of the left chelipod. Carapace width $(\mathrm{CW})$ was used as the reference dimension. Excepting the height of the propodus of juveniles and the upper length of the chelae of females whose relative growth was described by a linear model, all variables were better described by the traditional potential model. Results of the analysis showed that morphological changes detected during the ontogeny of A. patagoniensis are related to reproduction. The occurrence of transition points in the chelae and abdomen growth indicates that the species matures in Balneário Camboriú at 7,8-7,9 mm (males) and 7,9-8,3 mm (females) of carapace width. The same values were found also for the species in Cassino beach, Rio Grande do Sul $\left(32^{\circ} 13^{\prime} \mathrm{S}, 52^{\circ} 15^{\prime} \mathrm{W}\right)$, where the species is associated to another host, the callianassid shrimp Sergio mirim (Rodrigues, 1971). Therefore, it is suggested that the size at maturity of $A$. patagoniensis does not vary between hosts and latitude (at least between the studied limits), in spite of the differences observed in maximum attained sizes, fecundity and reproductive behavior of the two sites. KEY WORDS. Allometry, Brachyura, sexual maturity.
\end{abstract}

RESUMO. O crescimento relativo de Austinixa patagoniensis (Rathbun, 1918), simbionte do callianassídeo Callichirus major

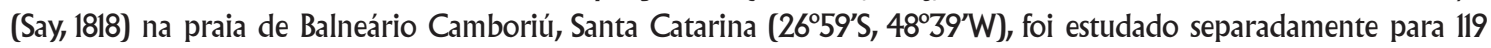
juvenis, 228 machos e 249 fêmeas. O método funcional de regressão foi utilizado para o estudo das seguintes variáveis: largura e comprimento da carapaça, largura máxima do abdome, altura máxima do própodo do quelípodo, comprimento inferior e superior total do própodo do quelípodo. A largura da carapaça (LC) foi adotada como medida de referência. Com exceção da altura do própodo do quelípodo de juvenis e do comprimento superior do própodo do quelípodo de fêmeas, que cresceram segundo o modelo linear, as demais variáveis estudadas foram melhor descritas pelo modelo potencial. A análise do crescimento relativo de $A$. patagoniensis permitiu observar que as diversas alterações morfológicas processadas ao longo da ontogenia desta espécie encontram-se relacionadas com fins reprodutivos. A presença de pontos de transição no crescimento da quela e do abdome, permitiu estimar o tamanho de maturação sexual de A. patagoniensis na praia de Balneário Camboriú em 7,8-7,9 mm e 7,9-8,3 mm (LC) para machos e fêmeas, respectivamente. Valores praticamente idênticos foram encontrados para a mesma espécie na praia do Cassino, Rio Grande do Sul ( $\left.32^{\circ} 13^{\prime} \mathrm{S}, 5^{\circ} 15^{\prime} \mathrm{W}\right)$, onde A. patagoniensis está associada ao também callianassídeo Sergio mirim (Rodrigues, 1971). Dessa forma, sugere-se que o tamanho de maturação da espécie não varia entre hospedeiros e no intervalo latitudinal analisado, apesar de terem sido detectadas diferenças no tamanho máximo atingido pelos organismos, assim como na sua fecundidade e no comportamento reprodutivo.

PALAVRAS CHAVE. Alometria, Brachyura, maturação sexual.

Revista Brasileira de Zoologia 22 (3): 784-792, setembro 2005 
Dados morfométricos têm sido largamente utilizados para estudos de crescimento relativo em crustáceos. Este grupo é particularmente atrativo para a realização destes estudos pois, além de possuir um exoesqueleto rígido que permite efetuar medidas acuradas, exibe grandes modificações nas relações morfométricas associadas normalmente ao dimorfismo e maturidade sexual (HARTNOLL 1982).

O estudo do crescimento relativo torna possível descrever de forma prática o desenvolvimento pós-embrionário de várias espécies de crustáceos (Tessier 1960). Neste grupo, o crescimento é caracterizado por uma série de fases, sendo que a transição entre as mesmas pode ser identificada através da presença de descontinuidades nas taxas alométricas de determinadas variáveis (HARTNOLL 1974). A natureza e a extensão destas descontinuidades variam, geralmente processando-se de forma mais abrupta durante o ingresso do organismo na puberdade (Hartnoll 1978).

Embora diversas técnicas para a determinação da maturidade sexual encontrem-se disponíveis (Совв \& CADDy 1989), a maioria despende muito tempo e envolve a danificação dos espécimes (Gore \& SotTo 1983). Por outro lado, estudos alométricos podem ser bastante eficientes e têm sido largamente utilizados com esta finalidade (e.g. Alves \& Pezzuto 1999, Lopes-Greco et al. 2000, Negreiro-Fransozo et al. 2003), pois existem partes do corpo que apresentam padrões fortes e consistentes de variação no nível de alometria associados a maturação das gônadas, notavelmente como a quela e o abdome de braquiúros (HARTNOLL 1982).

Os caranguejos da família Pinnotheridae apresentam um desenvolvimento pós-larval complexo, que envolve forte dimorfismo sexual e uma série de modificações morfológicas (CAMPOs 1993). No entanto, trabalhos que se dediquem exclusivamente ao estudo do crescimento relativo das espécies desta família são extremamente raros (Needham 1950, Alves \& Pezzuto 1999).

Austinixa patagoniensis (Rathbun, 1918) se distribui desde o Rio de Janeiro (Brasil) (Melo 1996) até o Golfo de San Matias (Argentina) (MANning \& Felder 1989). Nas praias arenosas brasileiras, esta espécie é caracteristicamente encontrada habitando a porção superior das galerias escavadas por crustáceos calianassídeos (Rodrigues \& SHImizu 1997). Embora constitua um dos principais componentes da macrofauna de praias arenosas, publicações que abordem a biologia e ecologia desta espécie são ainda muito escassos (Alves \& Pezzuto 1998a, b, Alves \& Rodrigues 2003).

Na praia do Cassino, litoral do Rio Grande do Sul $\left(32^{\circ} 13^{\prime}\right.$ S, $\left.52^{\circ} 15^{\prime} \mathrm{W}\right)$, A. patagonienis habita as galerias do calianassídeo Sergio mirim (Rodrigues, 1971), sendo que aspectos relacionados aos seus padrões de distribuição e dinâmica populacional foram investigados por Alves \& Pezzuto (1998a, b). Estudando o crescimento relativo da espécie na região, Alves \& Pezzuto (1999) estimaram o tamanho de maturação sexual da espécie.

Entretanto, СовB \& CADDY (1989) ressaltam que o tamanho de maturação sexual pode não ser constante para uma es- pécie ao longo de toda a sua distribuição geográfica. Tratandose de uma espécie simbionte, pode-se inferir ainda que possíveis diferenças nos hospedeiros utilizados também poderiam acarretar modificações nos parâmetros populacionais das espécies investigadas.

Sendo assim, este trabalho tem como objetivo principal verificar a possível existência de diferenças no tamanho de maturação de populações de $A$. patagoniensis residentes em diferentes latitudes e hospedeiros da costa sul do Brasil. Para tanto, descreve o crescimento relativo e estima o tamanho de maturação sexual da população de $A$. patagoniensis residente na praia de Balneário Camboriú (Santa Catarina) e associada ao callianassídeo Callichirus major (Say, 1818), comparando os resultados com aqueles obtidos por Alves \& Pezzuto (1999) para a mesma espécie na praia do Cassino (Rio Grande do Sul), onde a mesma ocorre associada a $S$. mirim.

\section{MATERIAL E MÉTODOS}

Os organismos utilizados no presente estudo foram coletados mensalmente das galerias de C. major, entre agosto de 1996 e julho de 1997, na praia de Balneário Camboriú, localizada no litoral centro-norte de Santa Catarina $\left(26^{\circ} 59^{\prime} \mathrm{S}, 48^{\circ}\right.$ $39^{\prime} \mathrm{W}$ ) e caracterizada como uma praia semi-protegida, composta por areia fina a muito fina e bem selecionada (Temme et al. 1997, Klein et al. 2002). Como instrumento de captura empregou-se uma bomba manual de sucção, acionada sobre 50 galerias de $C$. major selecionadas aleatoriamente no mediolitoral. O material extraído das galerias foi peneirado em malha de $0,5 \mathrm{~mm}$ de abertura e fixado em solução de formol a $4 \%$.

As análises foram efetuadas separadamente para 119 juvenis, 228 machos e 249 fêmeas de $A$. patagoniensis em função da possível existência de diferenças no crescimento relativo de determinadas variáveis atribuídas ao sexo (HARTNoll 1978, 1982). A determinação do sexo foi efetuada com base na análise dos pleópodos, sendo classificados ainda como juvenis aqueles organismos que não apresentaram a forma destas estruturas diferenciada em machos e fêmeas.

A largura (LC) e comprimento (CC) da carapaça, largura do abdome (medida entre os segmentos quatro e cinco) (LA), altura máxima do própodo do quelípodo esquerdo (AQ), e comprimento inferior total (incluindo o dedo fixo) (CIQ) e superior (CSQ) do própodo do quelípodo esquerdo foram medidos sob microscópio estereoscópico com auxílio de ocular micrométrica. A precisão utilizada foi de $0,1 \mathrm{~mm}$ para as três primeiras dimensões e 0,05 mm para as três últimas. No caso de organismos juvenis todas as dimensões foram medidas com $0,05 \mathrm{~mm}$ de precisão. Cabe destacar que as mensurações foram realizadas apenas sobre a quela esquerda em função da inexistência de dimorfismo entre os quelípodos de um mesmo indivíduo. A largura da carapaça (LC) foi utilizada como medida de referência.

Todas as análises foram efetuadas seguindo a mesma metodologia utilizada por Alves \& Pezzuto (1999) com o em- 
prego do programa REGRANS (PezzuTo 1993), que incorpora diversas técnicas de análise bivariada de crescimento relativo e permite uma análise comparativa dos dados. O método de regressão adotado foi o funcional (RICKER 1973).

Regressões lineares e potenciais foram calculadas para cada dimensão considerada, adotando-se posteriormente aquela com melhor ajuste, o qual foi verificado através dos menores valores obtidos para a soma dos produtos dos resíduos em x e y (SPXY) como sugerido por Pezzuto (1993).

A identificação de alterações no nível de alometria ao longo da ontogenia, foi realizada através de uma sub-rotina específica do programa REGRANS, a qual detecta de forma estatística a existência de pontos de transição ao longo do crescimento das variáveis analisadas (Pezzuto 1993).

As regressões obtidas por Alves \& Pezzuto (1999) para o crescimento relativo da população de $A$. patagoniensis residente na praia do Cassino $\left(32^{\circ} 13^{\prime} \mathrm{S} ; 52^{\circ} 15^{\prime} \mathrm{W}\right)$ foram utilizadas para fins comparativos.

Com o objetivo de comparar os aspectos reprodutivos das duas populações face a seus padrões de crescimento relativo, foram observados o tamanho máximo de machos e fêmeas, o tamanho mínimo de fêmeas ovígeras, o número médio de ovos produzidos por fêmea e a extensão do período reprodutivo. A fecundidade foi obtida mediante contagem direta dos ovos de 87 e 21 fêmeas em Balneário Camboriú e Cassino, respectivamente. Já os períodos reprodutivos foram identificados a partir do cálculo das porcentagens de fêmeas ovígeras nas amostras, em cada mês de coleta. Os dados provenientes do Rio Grande do Sul foram obtidos de Alves \& Pezzuto (1998b).

\section{RESULTADOS}

Através da análise do crescimento relativo foi possível detectar diferenças nas taxas de crescimento de certas dimensões corpóreas entre machos e fêmeas de $A$. patagoniensis.

Com exceção da altura do própodo do quelípodo de juvenis (AQ) e do comprimento superior do própodo do quelípodo de fêmeas (CSQ), que cresceram segundo o modelo linear, as demais variáveis estudadas foram melhor descritas pelo modelo potencial (Tab. I).

O comprimento da carapaça de juvenis, machos e fêmeas de $A$. patagoniensis apresentou crescimento alométrico negativo $(\mathrm{v}<1)$ sem a presença de pontos de transição (Tab. I, Figs 1 e 2). As declividades das curvas de machos e fêmeas não foram significativamente diferentes entre $\mathrm{si}(\mathrm{t}=1,1 ; \mathrm{p}>0,05)$.

O crescimento da largura do abdome de juvenis (Fig. 3) e machos (Fig. 4) apresentou alometria positiva ( $\mathrm{v}>1$ ) e levemente negativa, respectivamente, sem a presença de pontos de transição (Tab. I). Para fêmeas, o crescimento foi alométrico positivo com um ponto de inflexão em 5,4 mm (LC), onde um acréscimo na taxa de crescimento foi observado, e uma descontinuidade em 7,9 mm (LC) (Fig. 4), onde ocorreu um aumento brusco na largura do abdome (representado pelo aumento da elevação) e posterior diminuição de sua taxa de cres- cimento a qual se manteve, contudo, sob alometria positiva (Tab. I). Com relação a esta descontinuidade cabe ressaltar que, embora o programa REGRANS, em decorrência das especificações de sua rotina, tenha identificado um ponto de transição em 7,9 mm (LC), pode-se notar, através de uma análise visual dos dados da figura 4, que a descontinuidade ocorreu em um intervalo de tamanho variável entre 7,9 e 8,3 mm (LC).

A altura máxima do própodo do quelípodo apresentou crescimento linear sem pontos de transição em juvenis (Fig. 5), enquanto em machos e fêmeas (Fig. 6) exibiu um crescimento potencial. Em machos, um ponto de inflexão ocorreu em 7,9 mm (LC), a partir do qual observou-se uma aumento pronunciado na taxa de crescimento desta dimensão (Tab. I). Em fêmeas, um ponto de inflexão foi observado em 5,3 mm (LC), dimensão a partir da qual o crescimento passou de alométrico positivo para alométrico negativo em conseqüência de uma diminuição na taxa de crescimento da altura do própodo do quelípodo (Tab. I).

O comprimento inferior total do própodo do quelípodo apresentou crescimento isométrico em juvenis (Fig. 7) e machos (Fig. 8), e alometria levemente negativa em fêmeas (Fig. $8)$, sendo que em nenhum dos casos detectou-se pontos de transição (Tab. I).

A ausência de pontos de transição também foi verificada no crescimento do comprimento superior total do própodo do quelípodo em juvenis (Fig. 9), o qual exibiu alometria positiva. O mesmo não ocorreu em machos e fêmeas (Fig. 10), os quais apresentaram pontos de inflexão em 7,8 mm (LC) e $8,1 \mathrm{~mm}$ (LC), respectivamente (Tab. I). Em machos (Fig. 10), a alometria positiva observada no crescimento desta dimensão sofreu um acréscimo substancial após o ponto de inflexão. Em fêmeas, por sua vez, o crescimento foi linear com diminuição na taxa de crescimento após o ponto de inflexão (Fig. 10).

\section{DISCUSSÃO}

O presente estudo permitiu observar que, de modo geral, as alterações morfológicas processadas ao longo da ontogenia de $A$. patagoniensis encontram-se relacionadas com a aquisição da maturidade sexual.

A tabela II mostra as regressões obtidas por Alves \& Pezzuto (1999) para a população do Rio Grande do Sul. Com exceção do comprimento da carapaça de juvenis, que apresentou um ponto de inflexão ao longo de seu crescimento, as demais variáveis estudadas apresentaram padrão de crescimento semelhante ao verificado para a espécie em Balneário Camboriú (Tab. I).

Variações na forma da carapaça, entre machos e fêmeas de $A$. patagoniensis, não foram detectados no presente estudo, o que é corroborado pela similaridade verificada nas taxas de crescimento da relação CC/LC entre os sexos. Este mesmo padrão foi verificado para diversas espécies de braquiúros (HaRTNOLL 1982), para as quais a taxa de crescimento pode variar de isométrica (Pinheiro \& Fransozo 1993) a alométrica positiva (Abello et al. 1990). Estes resultados foram também muito similares aos 

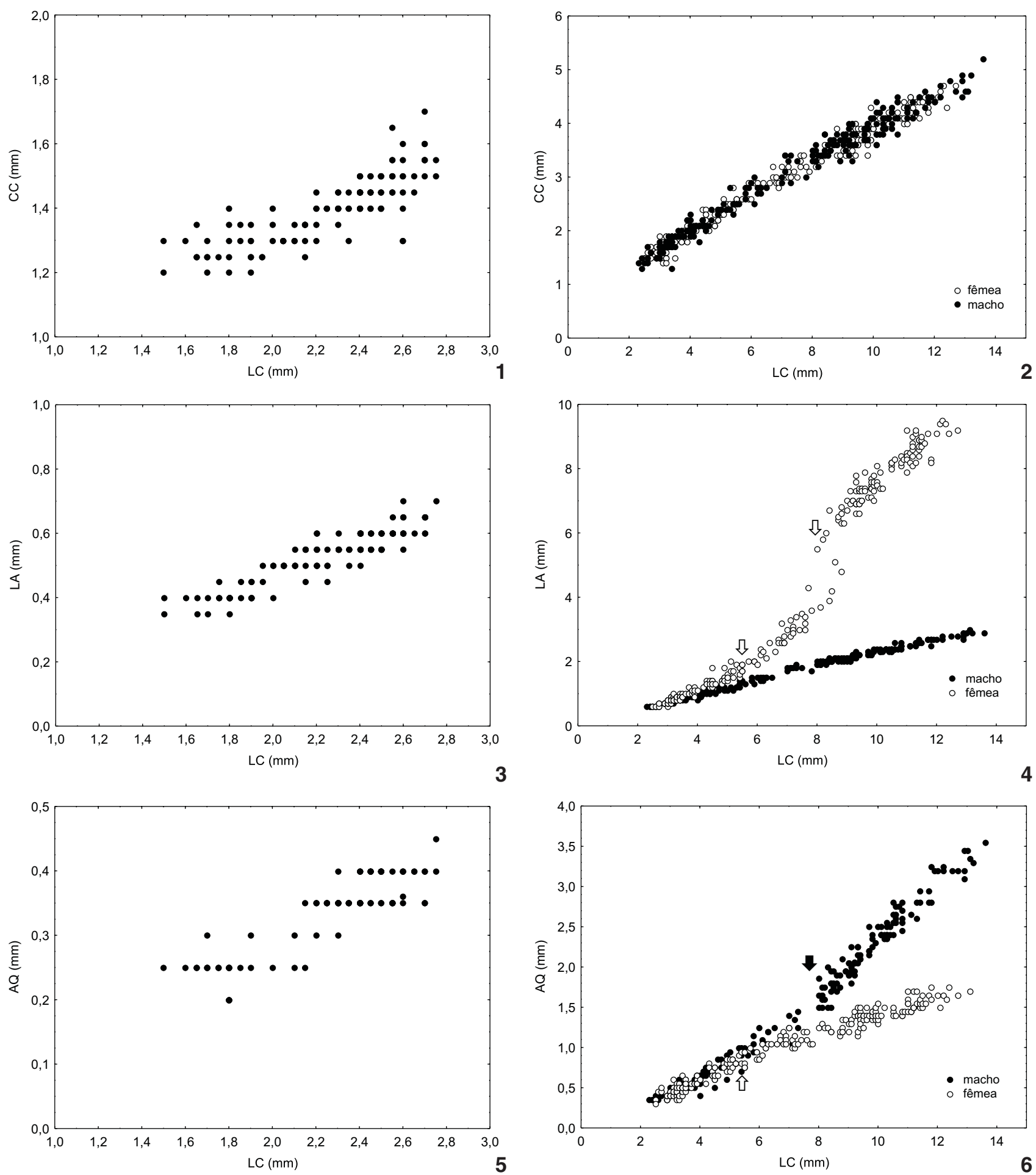

Figuras 1-6. Austinixa patagoniensis, relação entre: comprimento (CC) e largura da carapaça (LC) de juvenis (1), machos e fêmeas (2); largura do abdome (LA) e a largura da carapaça (LC) de juvenis (3), machos e fêmeas (4); altura máxima do própodo do quelípodo esquerdo (AQ) e a largura da carapaça (LC) de juvenis (5), machos e fêmeas (6), coletados das galerias de Callichirus major no mediolitoral da praia de Balneário Camboriú (Santa Catarina). A seta indica a presença de um ponto de transição. 

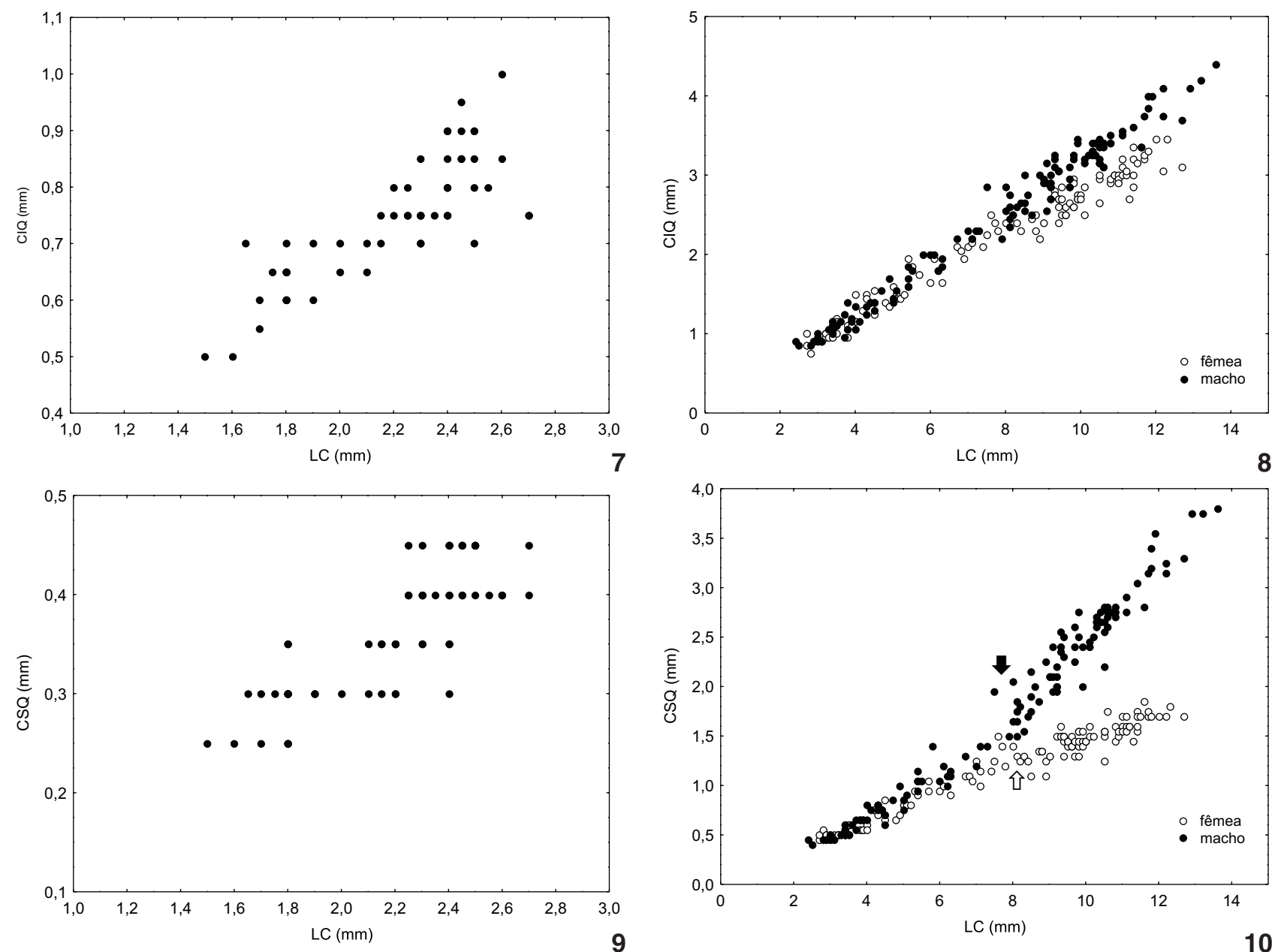

Figuras 7-10. Austinixa patagoniensis, relação entre: comprimento inferior do própodo do quelípodo esquerdo (CIQ) e a largura da carapaça (LC) de juvenis (7), machos e fêmeas (8); comprimento superior do própodo do quelípodo esquerdo (CSQ) e a largura da carapaça (LC) de juvenis (9), machos e fêmeas (10), coletados das galerias de Callichirus major no mediolitoral da praia de Balneário Camboriú (Santa Catarina). A seta indica a presença de um ponto de transição.

encontrados por Alves \& Pezzuto (1999) para a mesma espécie no Rio Grande do Sul (Tab. II), onde, contudo, os valores de $u$ e $v$ mostraram-se um pouco inferiores aos do presente estudo. Alves \& Pezzuto (1999) observaram ainda a presença de um ponto de transição no crescimento do comprimento da carapaça de organismos juvenis, o qual associaram a existência de uma muda crítica a partir da qual um crescimento maior na largura da carapaça em relação ao seu comprimento é verificado. Como estes autores trabalharam com uma precisão de $0,01 \mathrm{~mm}$ na medição dos organismos, a ausência de ponto de transição para a relação CC/LC de juvenis de $A$. patagoniensis no presente trabalho pode constituir um efeito da menor precisão utilizada para a mensuração dos organismos (0,05 mm).

A largura do abdome apresentou um forte dimorfismo sexual, sendo bem menor em machos do que em fêmeas da espécie. O crescimento da largura do abdome de machos de $A$. patagoniensis seguiu o padrão observado para a maioria dos caranguejos braquiúros, os quais não apresentam crescimento alométrico positivo para esta dimensão. Em braquiúros, o crescimento relativo do abdome de machos é geralmente isométrico ao longo de sua ontogenia (Hartnoll 1974, Pohle \& Telford 1982, Clayton 1990, Haefner 1990, Pinheiro \& Fransozo 1993) ou alométrico negativo como encontrado para a população de A. patagoniensis existente no litoral do Rio Grande do Sul (Alves \& Pezzuto 1999) (Tab. II). Isto porque, nos caranguejos, o abdome de machos é utilizado apenas para proteger e sustentar o primeiro par de pleópodos, os quais são utilizados como órgãos intromitentes durante a cópula (HARTNOLL 1982). 
Tabela I. Estatísticas para as regressões funcionais do comprimento da carapaça (CC), largura do abdome (LA), e altura (AQ), comprimento inferior (CIQ) e comprimento superior (CSQ) do própodo do quelípodo esquerdo, em relação à largura da carapaça (LC), calculadas separadamente para juvenis, machos e fêmeas de Austinixa patagoniensis, em Balneário Camboriú, e considerando os pontos de transição determinados pelo programa REGRANS (Pezzuto 1993). (u) Elevação, (v) declividade, (I.C.) intervalo de confiança de $95 \%$ para v. Segmento - intervalo de pontos da largura da carapaça $(\mathrm{mm})$ considerado nas regressões. Total - equação calculada para a totalidade dos dados, na ausência de pontos de transição significativos. Os pontos de transição que definem os segmentos na largura da carapaça estão expressos em milímetros.

\begin{tabular}{|c|c|c|c|c|c|c|c|}
\hline Dimensão & Sexo & Modelo & Segmento & $\mathrm{u}$ & $\mathrm{v}$ & I.C. & $r$ \\
\hline & Juvenil & Potencial & Total & 0,935 & 0,497 & $(0,444-0,551)$ & 0,813 \\
\hline \multirow[t]{4}{*}{$\mathrm{CC}$} & Macho & Potencial & Total & 0,709 & 0,737 & $(0,724-0,751)$ & 0,989 \\
\hline & Fêmea & Potencial & Total & 0,737 & 0,727 & $(0,714-0,740)$ & 0,989 \\
\hline & Juvenil & Potencial & Total & 0,208 & 1,151 & $(1,080-1,227)$ & 0,919 \\
\hline & Macho & Potencial & Total & 0,258 & 0,953 & $(0,941-0,964)$ & 0,994 \\
\hline \multirow[t]{5}{*}{ LA } & Fêmea & Potencial & $<5,4$ & 0,141 & 1,506 & $(1,423-1,594)$ & 0,958 \\
\hline & & & $5,4-7,9$ & 0,042 & 2,175 & $(1,966-2,407)$ & 0,953 \\
\hline & & & $>7,9$ & 0,204 & 1,560 & $(1,410-1,726)$ & 0,853 \\
\hline & Juvenil & Linear & Total & $-0,096$ & 0,189 & $(0,173-0,208)$ & 0,885 \\
\hline & Macho & Potencial & $<7,9$ & 0,110 & 1,273 & $(1,187-1,365)$ & 0,978 \\
\hline \multirow[t]{4}{*}{$A Q$} & & & $>7,9$ & 0,063 & 1,564 & $(1,473-1,660)$ & 0,957 \\
\hline & Fêmea & Potencial & $<5,3$ & 0,124 & 1,141 & $(1,014-1,286)$ & 0,897 \\
\hline & & & $>5,3$ & 0,246 & 0,761 & $(0,722-0,801)$ & 0,959 \\
\hline & Juvenil & Potencial & Total & 0,338 & 1,005 & $(0,869-1,162)$ & 0,849 \\
\hline \multirow[t]{4}{*}{ CIQ } & Macho & Potencial & Total & 0,301 & 1,025 & $(0,999-1,051)$ & 0,990 \\
\hline & Fêmea & Potencial & Total & 0,342 & 0,912 & $(0,886-0,938)$ & 0,986 \\
\hline & Juvenil & Potencial & Total & 0,133 & 1,265 & $(1,097-1,459)$ & 0,833 \\
\hline & Macho & Potencial & $<7,8$ & 0,120 & 1,244 & $(1,151-1,344)$ & 0,963 \\
\hline \multirow[t]{3}{*}{ CSQ } & & & $>7,8$ & 0,056 & 1,640 & $(1,508-1,784)$ & 0,934 \\
\hline & Fêmea & Linear & $<8,1$ & $-0,066$ & 0,176 & $(0,164-0,189)$ & 0,974 \\
\hline & & & $>8,1$ & $-0,030$ & 0,150 & $(0,130-0,173)$ & \\
\hline
\end{tabular}

Ao contrário de machos, o abdome de fêmeas desempenha uma importante função, em conjunto com o esterno, na incubação e proteção dos ovos, apresentando freqüentemente um alargamento substancial na maturidade sexual (HARTNOLL 1982). Em pinoterídeos, descontinuidades no crescimento do abdome relacionados à maturidade sexual foram observadas para Dissodactylus mellitae (Rathbun, 1900) (Bell 1988) e D. primitivus (Pohle \& Telford 1982). Em Balneário Camboriú, a diferenciação da largura do abdome de fêmeas de $A$. patagoniensis começou a ocorrer em 5,4 mm (LC), e foi marcada por um aumento na taxa de crescimento em relação a largura da carapaça. Praticamente neste mesmo ponto teve início a diminuição do crescimento da altura do quelípodo, sugerindo que a partir deste tamanho, fêmeas começam a investir sua energia no crescimento do abdome em detrimento de estruturas reprodutivamente menos importantes. Da mesma forma, um brusco alargamento no abdome ocorreu entre 7,9 $\mathrm{mm}$ e 8,3 mm (LC), acompanhado simultaneamente por uma leve diminuição na taxa de crescimento do comprimento superior do quelípodo em 8,1 mm (LC). Este mesmo padrão foi verificado para as fêmeas de A. patagoniensis residentes na praia do Cassino, onde porém os pontos de inflexão e transição ocorreram em 4,6 mm e 8,0 mm (LC), respectivamente (Alves \& Pezzuto 1999) (Tab. II).

Segundo HarTnOLL $(1974,1978,1982)$, a muda pré-puberal é identificada por uma mudança no nível de alometria, enquanto a muda puberal, que marca o fim da fase imatura, é caracterizada por mudanças morfológicas bruscas. Sendo assim, os pontos de transição que delimitaram as diferentes fases de crescimento do abdome e quelípodo de fêmeas de $A$. patagoniensis, em 5,3 $\mathrm{mm}$ e 7,9-8,3 $\mathrm{mm}$ (LC), parecem representar o momento da muda pré-puberal e puberal, respectivamente. O tamanho da muda puberal é ainda corroborado pelo tamanho mínimo encontrado para fêmeas ovígeras que foi de 7,9 mm (LC) (Tab. III).

Torna-se necessário ressaltar que após o significativo aumento ocorrido na largura do abdome de fêmeas de $A$. patagoniensis em 7,9-8,3 mm (LC), um decréscimo na taxa de crescimento foi verificado, indicando que este tamanho passa a ser suficiente para o cumprimento das funções reprodutivas. 
Tabela II. Estatísticas para as regressões funcionais (modelo potencial) do comprimento da carapaça (CC), largura do abdome (LA), e altura do própodo do quelípodo (AQ), em relação a largura da carapaça (LC), calculadas separadamente para juvenis, machos e fêmeas de Austinixa patagoniensis, na Praia do Cassino, e considerando os pontos de transição determinados pelo programa REGRANS (PEZzUTO 1993); (u) elevação, (v) declividade, (I.C.) intervalo de confiança de 95\% para v, (r) coeficiente de correlação. Segmento - intervalo de pontos da largura da carapaça $(\mathrm{mm})$ considerado nas regressões. Total - equação calculada para a totalidade dos dados, na ausência de pontos de transição significativos. Os pontos de transição que definem os segmentos na largura da carapaça estão expressos em milímetros. Fonte: Alves \& Pezzuto (1999).

\begin{tabular}{|c|c|c|c|c|c|c|}
\hline Dimensão & Sexo & Segmento & $\mathrm{u}$ & $\mathrm{v}$ & I.C. & $r$ \\
\hline \multirow[t]{4}{*}{$\mathrm{CC}$} & Juvenil & $<1,91$ & 0,683 & 1,165 & $0,861-1,488$ & 0,561 \\
\hline & & $>1,91$ & 0,642 & 0,828 & $0,787-0,974$ & 0,757 \\
\hline & Macho & Total & 0,696 & 0,734 & $0,719-0,748$ & 0,985 \\
\hline & Fêmea & Total & 0,708 & 0,722 & $0,707-0,736$ & 0,987 \\
\hline \multirow[t]{5}{*}{ LA } & Juvenil & Total & 0,485 & 1,257 & $1,180-1,340$ & 0,901 \\
\hline & Macho & Total & 0,256 & 0,961 & $0,935-0,989$ & 0,968 \\
\hline & Fêmea & $<4,60$ & 0,207 & 1,173 & $1,121-1,228$ & 0,936 \\
\hline & & $4,60-8,00$ & 0,063 & 1,945 & $1,840-2,055$ & 0,967 \\
\hline & & $>8,00$ & 0,440 & 1,236 & $1,128-1,354$ & 0,949 \\
\hline \multirow[t]{5}{*}{$\mathrm{AQ}$} & Juvenil & Total & 0,108 & 1,293 & $0,998-1,675$ & 0,672 \\
\hline & Macho & $<7,75$ & 0,096 & 1,327 & $1,222-1,441$ & 0,941 \\
\hline & Fêmea & $>7,75$ & 0,028 & 1,952 & $1,540-2,474$ & 0,841 \\
\hline & & $<4,74$ & 0,144 & 0,989 & $0,805-1,214$ & 0,858 \\
\hline & & $>4,74$ & 0,203 & 0,783 & $0,679-0,905$ & 0,830 \\
\hline
\end{tabular}

Tabela III. Tamanho máximo de machos e fêmeas, tamanho mínimo de fêmeas ovígeras, fecundidade média e período reprodutivo de Austinixa patagoniensis nas praias de Balneário Camboriú (Santa Catarina) e Cassino (Rio Grande do Sul). Todas as medidas referem-se à largura da carapaça (mm). Dados referentes à população do Rio Grande do Sul obtidos em Alves \& Pezzuto (1998b).

\begin{tabular}{|c|c|c|c|c|c|}
\hline \multirow{2}{*}{ Localidade } & \multicolumn{2}{|c|}{ Tamanho máximo } & \multirow{2}{*}{$\begin{array}{l}\text { Tamanho mínimo } \\
\text { fêmeas ovígeras }\end{array}$} & \multirow{2}{*}{$\begin{array}{l}\text { Número médio ovos } \\
\text { (mínimo-máximo) }\end{array}$} & \multirow{2}{*}{ Período reprodutivo } \\
\hline & Macho & Fêmea & & & \\
\hline Balneário Camboriú & 13,6 & 13,1 & 7,9 & 1719 (933-2747) & Contínuo \\
\hline Cassino & 11,5 & 11,5 & 8,0 & $1529(830-2472)$ & Outubro a março \\
\hline
\end{tabular}

De acordo com Hartnoll (1978), a taxa de crescimento do abdome tende a decrescer assim que este atinge um tamanho funcional. Isto ocorre pois qualquer aumento desproporcional na largura do abdome, pode diminuir a eficiência do mecanismo de incubação de ovos, e criar dificuldades para o organismo caminhar (HARTNOLL 1982).

O dimorfismo dos quelípodos entre os sexos ocorre em diversas espécies de pinoterídeos (Manning \& Felder 1989, ZMARZly 1992). Em indivíduos adultos de A. patagoniensis, além de possuírem formatos distintos, os quelípodos de machos são mais robustos do que em fêmeas (Melo 1996).

Em fêmeas de $A$. patagoniensis, com exceção da primeira fase de crescimento da altura do própodo do quelípodo $(<5,3$ mm LC), que apresentou alometria positiva, as demais dimensões do quelípodo exibiram alometria negativa ou incremento linear, indicando um pequeno investimento no crescimento destas dimensões corpóreas.

Por outro lado, os quelípodos são intensamente utilizados por machos de braquiúros na defesa territorial, combates, e cortejos às fêmeas, o que faz com que estes obtenham um tamanho funcional na maturidade (HARTNOLL 1974, 1978, 1982, LEVINGTON 1995). Em machos de A. patagoniensis, o padrão de crescimento observado para as diferentes dimensões do quelípodo é condizente com aquele observado para a maioria das espécies de caranquejos braquiúros (Tessier 1960, Hartnoll 1982, Haefner 1985, COMEAU \& CONAN 1992). Excetuando o crescimento do comprimento inferior do quelípodo de machos de A. patagoniensis, que ocorreu de forma isométrica, as demais dimensões apresentaram uma forte alometria positiva. Na altura e comprimento superior do quelípodo, as taxas de crescimento intensificaramse significativamente a partir de 7,9 $\mathrm{mm}$ e 7,8 $\mathrm{mm}$ (LC), respectivamente, indicando a ocorrência de uma muda puberal. 
Com base no reconhecido papel do quelípodo e do abdome nas funções reprodutivas dos braquiúros (HARTNOLL 1982) e nas marcadas alterações nas taxas de alometria, observadas na altura do própodo do quelípodo de machos (7,9 mm LC), na largura do abdome de fêmeas (entre 7,9 e 8,3 mm LC), e no comprimento superior do própodo do quelípodo em machos (7,8 mm LC) e fêmeas ( $8,1 \mathrm{~mm} \mathrm{LC})$, assumiu-se como tamanho provável de maturação de $A$. patagoniensis o intervalo de tamanho compreendido entre 7,9-8,3 mm (LC) para fêmeas, e 7,8-7,9 mm (LC) para machos. Estes tamanhos são praticamente os mesmos observados para a mesma espécie na praia do Cassino (Alves \& Pezzuto 1999) (Tabs II e III), indicando que o tamanho de maturação sexual de $A$. patagoniensis não varia entre hospedeiros e na faixa de sua distribuição geográfica considerada, embora tenham sido observadas variações significativas no tamanho máximo dos organismos, na fecundidade e no comportamento reprodutivo nos dois locais (Alves \& Pezzuto 1998b) (Tab. III).

A determinação da maturidade pode constituir um problema na família Pinnotheridae. Em algumas espécies a cópula ocorre muito precocemente e o sêmem do macho é armazenado no receptáculo seminal de fêmeas juvenis até que estas cresçam e alcancem a maturidade sexual (AtKins 1958, CHRISTENSEN \& McDermott 1958, Pearce 1964, 1966, Kruczynski 1973), o que inviabiliza um eventual exame da presença de espermatóforos nos receptáculos seminais de fêmeas como método de determinação do tamanho de maturação. Por outro lado, as marcadas alterações nas taxas de alometria dos caracteres sexuais secundários verificados para as duas populações de A. patagoniensis consideradas neste estudo, demonstram a importância da análise do crescimento relativo para a determinação da maturidade em pinoterídeos.

\section{AGRADECIMENTOS}

Os autores agradecem a Luiz Fernando Diehl, diretor do Centro de Ciências Tecnológicas da Terra e do Mar (UNIVALI/ SC) durante a realização deste trabalho, pela disponibilização de seus laboratórios no período de triagem do material biológico. Ao Conselho Nacional de Desenvolvimento Científico e Tecnológico (CNPq) pela concessão de bolsa de estudos para o primeiro autor durante a realização do trabalho.

\section{REFERÊNCIAS BIBLIOGRÁFICAS}

Abello, P.; J.P. Pertierra \& D.G. Reid. 1990. Sexual size dimorphism, relative growth and handedness in Liocarcinus depurator and Macropipus tuberculatus (Brachyura: Portunidae). Scientia Marina, Barcelona, 54 (2): 195-202.

Alves, E.S. \& P.R. Pezzuto. 1998a. Dispersão de Pinnixa patagoniensis no mediolitoral da praia do Cassino, RS. Atlântica, Rio Grande, 20: 5-21.

Alves, E.S. \& P.R. Pezzuto. 1998b. Population dynamics of Pinnixa patagoniensis Rathbun, 1918 (Brachyura: Pinnotheridae) a symbiotic crab of Sergio mirim (Thalassinidea: Callianassidae) in Cassino Beach, Southern Brazil. Pubblicazioni della Stazione Zoologica di Napoli Marine Ecology, Viena, 19 (1): 37-51.

Alves, E.S. \& P.R. Pezzuto. 1999. Crescimento relativo de Austinixa patagoniensis (Rathbun, 1918) (Decapoda: Pinnotheridae), simbionte de Sergio mirim (Rodrigues, 1971) (Decapoda: Callianassidae) na praia do Cassino, RS. Nauplius, Rio Grande, 7: 83-92.

Alves, E.S. \& S.A. Rodrigues. 2003. Distributional patterns of Austinixa patagoniensis (Rathbun, 1918) (Decapoda: Pinnotheridae), a symbiotic crab of Callichirus major (Decapoda: Callianassidae), in Balneário Camboriú Beach, Santa Catarina, Brazil. Journal of Coastal Research, Proceedings of the Brazilian Symposium on Sandy Beaches: Morphodynamic, ecology, uses, hazards and management. Royal Palm Beach, Special Issue 35: 408-417.

AtкIns, D. 1958. British pea crabs (Pinnotheres). Nature, London, 181: 1087.

BeLL, J.L. 1988. Distribution and abundance of Dissodactylus mellitae Rathbun (Pinnotheridae) on Mellita quinquiesperforata (Leske) (Equinodermata). Journal of Experimental Marine Biology and Ecology, Groton, 117: 93-114.

CAmpos, E. 1993. Systematics and taxonomic remarks on Pinnotheres muliniarum Rathbun, 1918 (Crustacea: Brachyura: Pinnotheridae). Proceedings of the Biological Society of Washington, Washington, 106 (1): 92-101.

Christensen, A.M. \& J.J. Mcdermott. 1958. Life-history and biology of the oyster crab, Pinnotheres ostreum Say. Biological Bulletin, Woods Hole, 114: 146-179.

Clayton, D.A. 1990. Crustacean allometric growth: a case for caution. Crustaceana, Leiden, 58 (3): 270-290.

Совв, J.S. \& J.F. Caddy. 1989. The population biology of Decapods, p. 327-374. In: J.F. CADDY (Ed.). Marine invertebrate fisheries: their assessment and management. New York, John Wiley and Sons, 752p.

Comeau, M. \& G.Y. Conan. 1992. Morphometry and gonad maturity of male snow crab, Chionoecetes opilio. Canadian Journal of Fisheries and Aquatic Sciences, Ottawa, 49: 2460-2468.

Gore, R.H. \& L.E. SotTo. 1983. Studies on decapod Crustacea from the Indian River region of Florida. XXV. Carapacial and abdominal allometry in five species of subtropical parthenopid crabs (Brachyura, Parthenopidae). Crustaceana, Leiden, 44 (1): 1-22.

Haefner JR., P.A. 1985. Morphometry, reproduction, diet and epizoites of Ovalipes stephensoni Williams, 1976 (Decapoda: Brachyura). Journal of Crustacean Biology, Seminole, 5 (4): 658-672.

Haefner JR., P.A. 1990. Morphometry and size at maturity of Callinectes ornatus (Brachyura: Portunidae) in Bermuda. Bulletin of Marine Science, Miami, 46 (2): 274-286.

HarTNOLL, R.G. 1974. Variation in growth pattern between some secundary sexual characters in crabs (Decapoda, Brachyura). 
Crustaceana, Leiden, 27 (2): 131-136.

Hartnoll, R.G. 1978. The determination of relative growth in Crustacea. Crustaceana, Leiden, 34 (3): 281-293.

Hartnoll, R.G. 1982. Growth, p. 111-196. In: G. Abele (Ed.). The Biology of Crustacea - embryology, morphology and genetics. London, London Academic Press, 440p.

Klein, A.H.F.; L. Benedet Filho \& D.H. Schumacher. 2002. Shortterm beach rotation processes in distinct headland bay beach systems. Journal of Coastal Research, Royal Palm Beach, 18 (3): 442-458.

Kruczynski, W.L. 1973. The effect of the pea crab Pinnotheres maculatus Say in Bogue Sound, North Carolina. Biological Bulletin, Woods Hole, 145: 482-491.

Levington, J.S. 1995. Marine Biology: Function, Biodiversity and Ecology. New York, Oxford University Press, 420p.

Lopez-Greco, L.; J. Hernandez; J. Bolanos; E. Rodriguez \& G. Hernandez. 2000. Population features of Microphrys bicornutus Latreille, 1825(Brachyura, Majidae) from Isla Margarita, Venezuela. Hydrobiologia, Brussels, 439 (1-3): 151-159

Manning, R.B. \& D.L. Felder. 1989. The Pinnixa cristata complex in the Western Atlantic, with a description of two new species (Crustacea: Decapoda: Pinnotheridae). Smithsonian Contributions to Zoology, Washington, 473: 1-26.

Melo, G.A.S. 1996. Manual de identificação dos Brachyura (caranguejos e siris) do litoral brasileiro. São Paulo, Ed. Plêiade, FAPESP, 604p.

Needham, A.E. 1950. The form-transformation of the abdomen of the female pea-crab, Pinnotheres pisum Leach. Proceedings of Royal Society of London, London, 137 (886): 115-136.

Negreiros-Fransozo, M.L.; K. D. Colpo \& T. M. Costa. 2003. Allometric growth in the fiddler crab Uca thayeri (Brachyura, Ocypodidae) from a subtropical mangrove. Journal of Crustacean Biology, Seminole, 23 (2): 273-279.

Pearce, J.B. 1964. On reproduction in Pinnotheres maculatus
(Decapoda: Pinnotheridae). Biological Bulletin, Woods Hole, 127: 384.

Pearce, J.B. 1966. On Pinnixa faba and Pinnixa littoralis (Decapoda: Pinnotheridae) symbiotic with the clam, Tresus capax (Pelecypoda: Mactridae), p. 565-589. In: H. BARnes (Ed.). Some Contemporary Studies in Marine Science, London, Allen and Unwin.

PezzuTo, P.R. 1993. REGRANS: a "BASIC" program for an extensive analysis of relative growth. Atlântica, Rio Grande, 15: 93-105.

Pinheiro, M.A.A. \& A. Fransozo. 1993. Relative growth of the sperckerd swimming crab Arenaeus cribarius (Lamarck, 1818) (Brachyura: Portunidae) near Ubatuba, State of São Paulo, Brazil. Crustaceana, Leiden, 65 (3): 377-389.

Pohle, G. \& M. Telford. 1982. Post-larval growth of Dissodactylus primitivus Bouvier, 1917 (Brachyura: Pinnotheridae) under laboratory condition. Bulletin of Marine Science, Miami, 33 (2): 257-273.

RICKER, W.E. 1973. Linear regression in fishery research. Journal of Fisheries Research Board of Canada, Ottawa, 30: 409-434.

Rodrigues, S.A. \& R.M. Shimizu. 1997. Autoecologia de Callichirus major (Say, 1818). Oecologia Brasiliensis, Rio de Janeiro, 3: 155-170.

Temme, B.; A.H.F. Klein; J.L.B. Carvalho \& F.L. Diehl. 1997. Morphologic behaviour of Balneário Camboriú beach, Santa Catarina - Brazil: preliminary results. Notas Técnicas da Facimar, Itajaí, 1: 49-65.

Tessier, G. 1960. Relative growth, p. 537-560. In: T.M. Waterman (Ed.). The Physiology of Crustacea. New York, Academic Press, vol. 1, 670p.

ZMarzly, D.L. 1992. Taxonomy review of pea crabs in the genus Pinnixa (Decapoda: Brachyura: Pinnotheridae) occurring on the California shelf, with descriptions of two new species. Journal of Crustacean Biology, Seminole, 12 (4): 677-713.

Recebido em 15.III.2005; aceito em 25.VIII.2005. 